\title{
Testing of a Novel Web Browser Interface for the Chinese Market
}

\author{
Siu-Tsen Shen ${ }^{1}$, Stephen D. Prior ${ }^{2}$, and Kuen-Meau Chen ${ }^{3}$ \\ ${ }^{1}$ Department of Multimedia Design, National Formosa University, \\ 64 Wen-Hua Rd, Hu-Wei 63208, Taiwan \\ ${ }^{2}$ Department of Product Design and Engineering, Middlesex University, \\ London N14 4YZ, United Kingdom \\ ${ }^{3}$ Department of Industrial Design, National United University, \\ 1 Lien Da, Kung-Ching Li, Maioli 36003, Taiwan \\ shen31@hotmail.com, s.prior@mdx.ac.uk, tancred_0721@hotmail.com
}

\begin{abstract}
This paper compares the perspicacity, appropriateness and preference of web browser icons from leading software providers with those of a culturespecific design. This online study was conducted in Taiwan and involved 103 participants, who were given three sets of web browser icons to review, namely Microsoft Internet Explorer, Macintosh Safari, and culturally specific icons created using the Culture-Centred Design methodology. The findings of the study show that all three sets have generally high recognition rates, but that some icon functions (e.g. Go/Visit and Favourite) in all three sets have poor recognition rates and are considered inappropriate.
\end{abstract}

Keywords: web browser icons, icons, perspicacity, Chinese, culturalisation, user interface design.

\section{Introduction of the Growth of the Chinese Market}

The recently published $22^{\text {nd }}$ statistical survey report - 'Internet Development in China' (July 2008), states that there are approximately 84.7 million computer hosts and 253 million Internet users in China. This only amounts to a penetration rate of $19.1 \%$ of the population [6]. Even with this low rate, China has now overtaken the USA (230 million) in terms of the number of Internet users. The number of Internet users in China has grown by 347\% during the period (2000-2006) [11], and if as predicted, China continues to grow at a conservative estimate of $40 \%$ per annum (note that Chinese Internet users grew by 43 million in the first half of 2008), it will approach saturation ( $\approx 70 \%$ penetration) by 2012 (see Fig 1 ).

The average weekly surfing time of Chinese internet users is currently $19 \mathrm{hrs,}$, with the largest professional sector within the Chinese internet market being Students with 76 million users $(30 \%)$. It is therefore no surprise that the 18-24 age group has the highest number of internet users. However, only $3.9 \%$ of people over 50 yrs use the internet, the biggest reason for not using the internet is stated as 'Not having the necessary skill' $(43.3 \%)$. China clearly needs to reach out to this underutilized market.

N. Aykin (Ed.): Internationalization, Design, LNCS 5623, pp. 413-418, 2009.

(c) Springer-Verlag Berlin Heidelberg 2009 


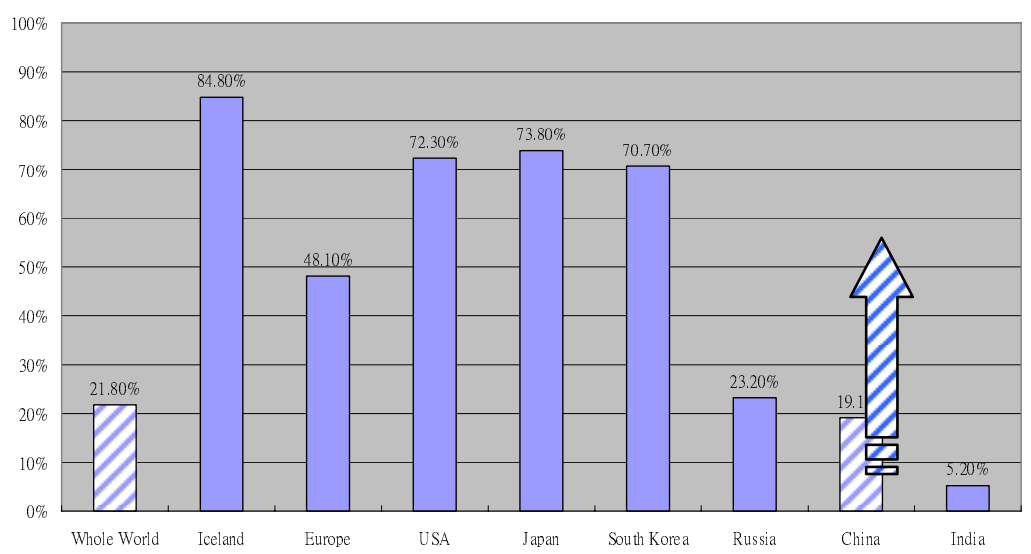

Fig. 1. Internet Penetration Rates [11]

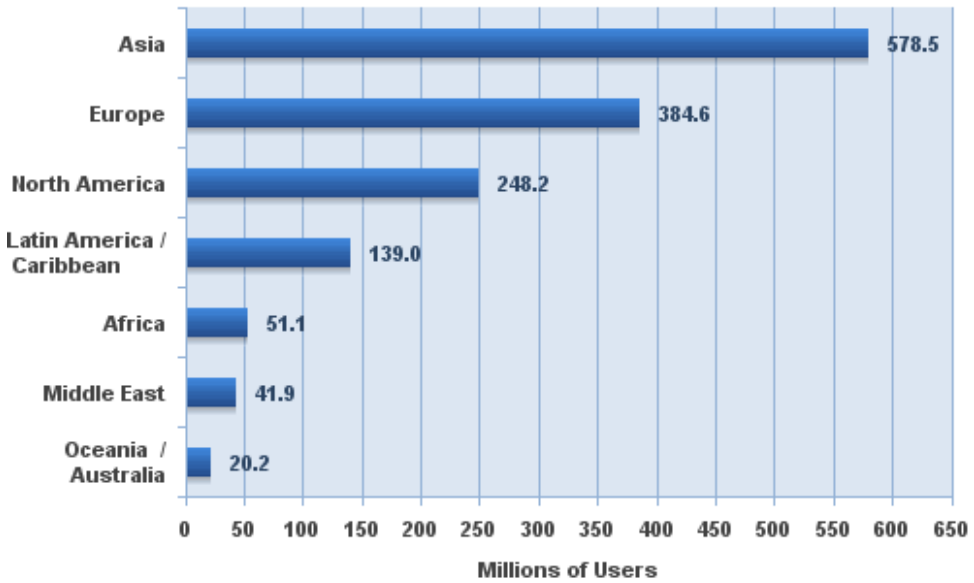

Fig. 2. Internet users by world region [10]

As of September 2008, 164 million people have downloaded the Maxthon browser since its launch in 2003. It has been reported that $14 \%$ of Chinese users have used the browser and $17 \%$ employ it for web searching through Baidu (the largest search engine in China) [12]. The reasons for its success are its customizable and innovative icon features, fast speed and the fact that it is built on top of the IE engine.

According to the August 2008 report from the Taiwanese Foreseeing Innovative New Digi-services (FIND) organisation, there were approximately 15.4 million Internet users $(67.2 \%$ penetration rate) in Taiwan, the most frequent use was for web browsing (71\%) [15].

Brandon [2] has suggested that a majority of internet users primarily speak languages other than English; Sun [14] has suggested that this could be as high as $70 \%$. It has also been reported that $75 \%$ of users in China and Korea prefer content in their 
own languages [9]. This mismatch highlights the need for more research and shows possible commercial potential.

\subsection{Cultural Aspect of Chinese Users}

It has been stated that Chinese users have better cognitive ability in terms of visual perception. Chinese characters are two-dimensional graphic symbols, which are made up of lexical and morphological elements, by comparison with one-dimensional, linear, alphabetical systems of Indo-European languages [5].

Choong and Salvendy [4] examined the impact of cultural differences on the cognitive abilities of American and Chinese users in terms of their performance time and errors with three different icon displays. Their results indicated that American subjects had better verbal ability with alphanumeric icon displays, whereas Chinese subjects had better visual distinction ability with pictorial icon displays, if both of subjects are not provided with combined modes.

Fang and Rau [8] reported that "The Chinese way of thinking tends to be synthetic, concrete and remains on the periphery of the visible world. The US way of thinking tends to be analytic, abstract and imaginative or beyond the realm of the immediately apprehended."

The purpose of this study was to investigate users' perspicacity, appropriateness and preference of web browser icons, and to compare the influence of gender, educational level and computer experience on these findings.

\section{Research Questions}

- How will the rapid growth in non-English speaking internet users affect the balance of power in terms of the development of the internet?

- How well can Chinese users associate IE 7.0 and Safari 3.0 web browser icons with their intended functions?

- Do Chinese users think that these representations are appropriate?

- Is it possible to design culturalised web browser icons for Chinese users?

- What form would these culturalised web browser icons take?

- Will Chinese users prefer to use culturalised web browser icons over the industry leading offerings from Microsoft Internet Explorer and Macintosh Safari?

Does the gender, educational level, level of computer experience or computer platform of the user play a role in determining any of the above factors?

\section{Research Methods}

The web browser icons chosen for this study were taken from Internet Explorer 7.0 and Safari 3.0, since Microsoft IE and Macintosh Safari are the two most frequently used PC and Mac web browser platforms. We then compared these with CultureCentred Design (CCD) (culturally specific) icons that aim to differentiate from standardised ones, and have been designed specifically for Chinese users [13]. Eight basic 


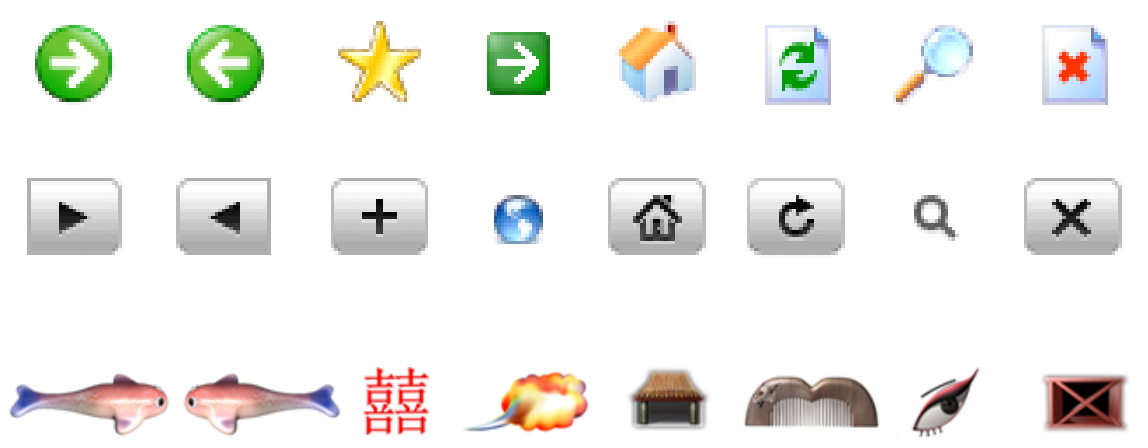

Fig. 3. Selection of IE 7.0, Safari 3.0, and CCD web browser icons

icon functions were selected from each of the latest IE, Safari, and CCD web browsers, i.e. Forward, Backward, My Favourite, Go/Visit, Home, Refresh, Search, and Stop. The order of these when presented to participants was deliberately mixed up to prevent guessing by test subjects.

The experiment was conducted online in Jan/Feb 2008 at the College of Art and Humanities, National Formosa University (NFU). The duration of the experiment was approximately 20 minutes which included tests for iconic perspicacity, appropriateness, and preference. Participants had to complete each page in order to continue to the following experimental page. No feedback or results were presented to the participant at the end of the test. There were a total of 103 undergraduate students (52 male, 51 female) involved in this online experiment through a website hosted by the department.

There were four categories as independent variables: (a) gender: males vs. females; (b) educational background: high school vs. college vs. university vs. graduate school; (c) level of computer experience: <2 yrs vs. 3-4 yrs vs. 5-6 yrs vs. 7-8 yrs vs. >9 yrs; and (d) the participants' regular computer platform: $P C$ vs. Mac. These four variables were tested independently to evaluate overall usability. The dependent variables were the usability measured by icon perspicacity, icon appropriateness, and icon user preference. Other relevant knowledge of the participants was shown by the use of the mean and standard deviation for qualitative analysis of icon perspicacity, appropriateness, and preference.

\section{Discussion of the Results}

Within this study a comparative experimental evaluation with 103 participants has been conducted using an online resource. The results of this study support the theory that Microsoft's Internet Explorer has successfully globalised non-English speaking internet users within Taiwan.

In terms of perspicacity, most users could easily associate the web browser icons with their intended functions. However, there were several usability problems reported with the IE 7.0 Go/Visit and Search icons. The Apple Safari web browser icons also caused several problems for users, of particular note being the Favourite, Go/Visit and Refresh icons. 
In terms of appropriateness, the participants felt that several of the IE icons were either highly inappropriate or inappropriate; in order of severity these were: Go/Visit (38\%), Favourite (30\%), Stop (27\%), and Forward (25\%). For the Apple Safari icons, participants felt that several icons fell into the categories of either highly inappropriate or inappropriate; in order of severity these were: Go/Visit (62\%), Favourite (52\%), Forward (27\%), Backward (25\%), and Refresh (25\%).

In order to gauge the level of support amongst Chinese computer users for a web browser containing specifically designed culturalised icons, we compared icons developed using the Culture-Centred Design methodology with those of IE 7.0 and Safari 3.0. Analysis of our results shows that the CCD icons had perspicacity rates, which were almost equivalent to those of IE 7.0 and Safari 3.0, however, when we analysed the data on appropriateness, it was clear that several of these icons had high levels of either highly inappropriate or inappropriate: Go/Visit (69\%), Refresh (65\%), Favourite (62\%), Forward (48\%), Backward (48\%), Stop (44\%), Search (40\%), and Home (38\%). To some extent, this is comprehensible due to the high levels of PC users $(98 \%)$ amongst the participants. However, we intend to further develop these to lower these levels in line with those of IE.

\section{Conclusions}

The Chinese netizen community is expanding rapidly and has recently overtaken the USA to become the largest Internet user base in the world. By 2012, we estimate that there could be over 900 million Internet users in China alone. However, having conducted a thorough literature review, we have found very few citations with regards to web browser icon developments specifically for Chinese users.

Of course it is true that Chinese culture is somewhat different between mainland China, Hong Kong Chinese and Taiwan Chinese, etc. However, in so far as they all communicate using a common ideographic language and share a similar cultural hegemony, we can consider them as a common group with shared cultural markers.

Web browser icons should be intuitive, associative and easy to navigate, in supporting the comprehensibility of Chinese web users. With the rapid growth of usage of computers and the Internet, designers need to be culturally-sensitive to specific users needs $[1,3,7,16]$.

The CCD icons used in this testing have been developed over several years, using Taiwanese participants, we believe that these show promise as alternative browser icons to both IE 7.0 and Safari 3.0. We fully accept that several of these require further enhancements to increase their perspicacity and appropriateness.

The market for web browsers is highly competitive and with the recent introduction of Google's Chrome it is getting ever more crowded. The level of acceptability and preference for Chinese users to use culturalised web browser icons over the traditional offerings from Internet Explorer and Safari remains challenging.

Icon preference testing results clearly show that the vast majority of participants prefer IE 7.0 icons over Safari 3.0 icons; and Safari 3.0 icons over CCD icons. These results are irrespective of gender and educational level. Again, to some extent, this is predictable given the high level of PC (IE) users. 
The results of this study provide a solid foundation for future development of all web browser icons. We believe that even the most successful browser, i.e. Internet Explorer can be improved by remodelling their Go/Visit and Favourite icons.

The strength of an iconic representation lies in the user's ability to recognise and interpret its functionality when taken out of context. Further issues that we would like to explore include a $2 \mathrm{D}$ versus $3 \mathrm{D}$ comparison, use of size, colour. In future we would also seek to balance user groups in terms of PC/Mac users, Experience Levels and Educational Levels.

\section{References}

1. Barber, W., Badre, A.: Culturability: The Merging of Culture and Usability. In: Proceedings of the 4th Human Factors and the Web Conference (1998)

2. Brandon, D.: Localization of web content. Journal of Computing in Small Colleges 17(2), 345-358 (2001)

3. Bourges-Waldegg, P., Scrivener, S.: Meaning, the Central Issue in Cross-Cultural HCI Design. Shared values and shared interfaces: The role of culture in the globalisation of human-computer systems. Interacting with Computers: the interdisciplinary journal of human-computer interaction 9(3), 287-309 (1998)

4. Choong, Y.Y., Salvendy, G.: Design of Icons for use by Chinese in Mainland China. Interacting with Computers 9(4), 417-430 (1998)

5. Chu, K.: The Cognitive Aspects of Chinese Character Processing. In: Chang, T.S.I., Ligomenides, P.A. (eds.) Visual Language, pp. 349-392. Plenum Press, New York (1986)

6. CNNIC. Statistical Survey Report on the Internet Development of China (2008), http://www.cnnic.net.cn/en/index/00/index.htm (cited September 7, 2008)

7. Every, D.K.: Innovation: Desktop Metaphor (Netscape 4.7) (1999),

http: / /www. mackido.com/Innovation/Desktop.html (cited November 13, 2001)

8. Fang, X., Rau, P.: Cultural differences in design of portal sites. Ergonomics 46(1-3), 242254 (2003)

9. Ferranti, M.: From Global to Local. In: Infoworld (1999)

10. Miniwatts. Internet Users by World Region (www) (2008), http: / /www. internetworldstats. com/stats.htm (cited September 7, 2008)

11. Miniwatts: Internet World Stats (August 14, 2008), http: / / www. internetworldstats.com/ (cited August 14)

12. Olsen, S.: Maxthon: China's hip browser (June 22, 2006), http://news .com.com/ (cited August 13, 2006)

13. Shen, S.T., Woolley, M., Prior, S.D.: Towards culture-centred design. Interacting with Computers 18(4), 820-852 (2006)

14. Sun, H.: Building a culturally-competent corporate web site: An exploratory study of cultural markers in multilingual web design. In: SIGDOC 2001, ACM, Santa Fe, New Mexico, USA (2001)

15. TWNIC: The report of the use of broadband networking in Taiwan (July 2008), http: / /www. twnic. net/NEWS / 1085 . bin (cited September 7, 2008)

16. Yeo, A.W.: Are usability assessment techniques reliable in non- western cultures? (August 23, 2000),

http://www.cityu.edu.hk/is/ejisdc/vol3/v3r1.pdf (cited 2001) 